\title{
Bebidas ancestrales y tradicionales de Colombia (Región del Pacífico)*
}

\section{Ancestral and traditional Colombian drinks (Pacific región)}

\author{
MEDARDO SÁENZ MONSALVE ${ }^{5}$ \\ (10) https://orcid.org/0000-0002-6462-4985
}

Artículo de investigación

Recepción: 04 de mayo de 2020

Aceptación: 24 de junio de 2020

Cómo citar este artículo

Sáenz Monsalve, M. 2020). Bebidas ancestrales y tradicionales de Colombia (Región del Pacífico). Sosquua. Revista Especializada en Gastronomía 2(1), pp. 57-66 Recuperado a partir de: http://cipres.sanmateo.edu.co/index.

php/sosquua

Reconocimiento-SinObraDerivada 4.0 Internacional (CC BY-NC-ND)

\footnotetext{
* El autor agradece a Laura Daniela Clavijo Castañeda, Laura Camila Rozo Callejas, Yesid Mateo Pardo Cruz, Valentina Cárdenas Pedraza, María Isabel Cuevas Riaño y Sebastián Felipe Borda Vargas, estudiantes en el programa de gastronomía de la Fundación Universitaria San Mateo (Bogotá), quienes colaboraron en la ejecución del proyecto de investigación.

${ }^{5}$ Docente investigador, Fundación Universitaria San Mateo. Correo electrónico: msaenz@sanmateo.edu.co
} 


\section{Resumen}

La gastronomía como contexto de desarrollo socio cultural, ha definido un gran avance en muchas culturas a nivel nacional, esto representado en los diferentes ingredientes y productos agrícolas de cada región, dentro de estos se encuentran una gran variedad de frutos tanto reconocidos como exóticos con los cuales se elaboran múltiples bebidas, sumado a las bebidas alcohólicas que se elaboran de manera tradicional y asimismo industrializadas, partiendo de la ubicación geográfica de cada región. Por medio de la presente indagación se busca identificar y generar un material pedagógico para el reconocimiento de preparaciones ancestrales en bebidas de la región del Pacifico. Como fuente de formación profesional del gastrónomo colombiano, uno de los ejes de valor es brindar el reconocimiento por medio de las bebidas buscando evidenciar la cultura de las regiones mediante el análisis de sus ingredientes y técnicas de elaboración. Mediante una metodología etnográfica se buscó establecer los principales contextos socio-culturales que se generan en la costa pacífica y mediante un análisis cualitativo se buscó contrastar las preparaciones ancestrales con el reconocimiento de patrimonio inmaterial dentro del campo de la gastronomía.

Palabras clave: bebidas; tradición; cultura; patrimonio; región Pacífica.

\section{Abstract}

Gastronomy as a context of socio-cultural development has defined a breakthrough in many cultures at the national level. This represented in the different ingredients and agricultural products of each region. These are a wide variety of fruits both recognized and exotic with the which are made multiple beverages, added to alcoholic beverages that are made in a traditional and industrialized way, based on the geographical location of each region. Through this investigation, the aim is to identify and generate a pedagogical material for the recognition of ancestral preparations in beverages from the Pacific region. As a professional training source for the Colombian gourmet, one of the axes of value is to provide recognition through drinks seeking to highlight the culture of the regions by analyzing their ingredients and production techniques. Through an ethnographic methodology, we sought to establish the main socio-cultural contexts that are generated on the Pacific coast, and by means of a qualitative analysis, we sought to contrast ancestral preparations with the recognition of intangible heritage within the field of gastronomy.

Keywords: drinks; tradition; culture; legacy; Pacific region. 


\section{Introducción}

El pacífico colombiano es una de las regiones con mayor biodiversidad del país, con ingredientes, técnicas, rituales de gran valor cultural, por ello estas cocinas poseen tesoros gastronómicos para explorar; los cuales con el presente trabajo investigativo se expone gran parte de las bebidas tradicionales de la región, como lo son las alcohólicas y no alcohólicas, con el fin de dar a conocer no solo su preparación sino también gran parte de su historia.

La gran mayoría de estas bebidas tradicionales están presentes en festivales gastronómicos y de tradición cultural como lo es el Petronio Álvarez. Por medio de visita a este festival, y en el complemento con indagaciones se realizó un gran aporte al desarrollo de la muestra de las bebidas tradicionales. Así se generaron aportes socioeconómicos a cada familia dedicada a la elaboración de dichas bebidas.

Dentro de los contextos de investigación realizados se encontró la tradición, producción, comercialización y consumo de las bebidas artesanales alcohólicas y no alcohólicas de la región pacífica y de las bebidas no alcohólicas que generan un proceso de consumo alto debido a la ubicación geográfica con sus altas temperaturas estableciendo una necesidad de aclimatación generada por los frutos cítricos y sus derivados.

Es importante resaltar que estas bebidas tiene un gran influencia de la cultura africana, los cuales están orientados en representar su historia mediante el paso de los españoles por el territorio nacional, evidenciado tanto en la generación de historias, música, pintura y elementos gastronómicos que acompañan el desarrollo de la dieta de sus pobladores como de los extranjeros (Chavez, C. 2012 p.13).

Las bebidas ancestrales de la región pacifica son tradición, cultura y sostenimiento económico de muchas familias. Estas bebidas se han caracterizado porque en su mayoría son afrodisiacas de acuerdo con preceptos y generalidades de las creencias afro descendientes y beneficiosas para la salud realizadas por medio de rituales que son adoptadas por medio de la elaboración con adición de hierbas medicinales. 


\section{Metodología}

Mediante elementos de desarrollo etnográfico se buscó varias bebidas a trabajar por parte del equipo, organizando un grupo de bebidas alcohólicas y un grupo de bebidas no alcohólicas, para la generación de varios productos pedagógicos, los cuales buscaban describir la elaboración directa de las bebidas de acuerdo con los procesos básicos y materias primas base que se encuentran en los mercados nacionales.

En el desarrollo de la indagación las entrevistas se enfocaron a portadoras de tradición que representan la memoria viva de la cultura del pacífico colombiano, sumado a la presentación de sus saberes y desarrollos históricos evidenciados en el trabajo realizado por el Ministerio de Cultura (Granados, 2013, p. 33). Dentro de las técnicas metodológicas de investigación se desarrolló la visita al festival Petronio Álvarez. El objetivo fue buscar el producto a describir directamente en la zona; así como el encuentro tradicional de portadores de saberes de la región Pacífica y en el centro de la producción y presentación de las bebidas.

Figura 1. Bebidas alcohólicas y no alcohólicas del Pacífico

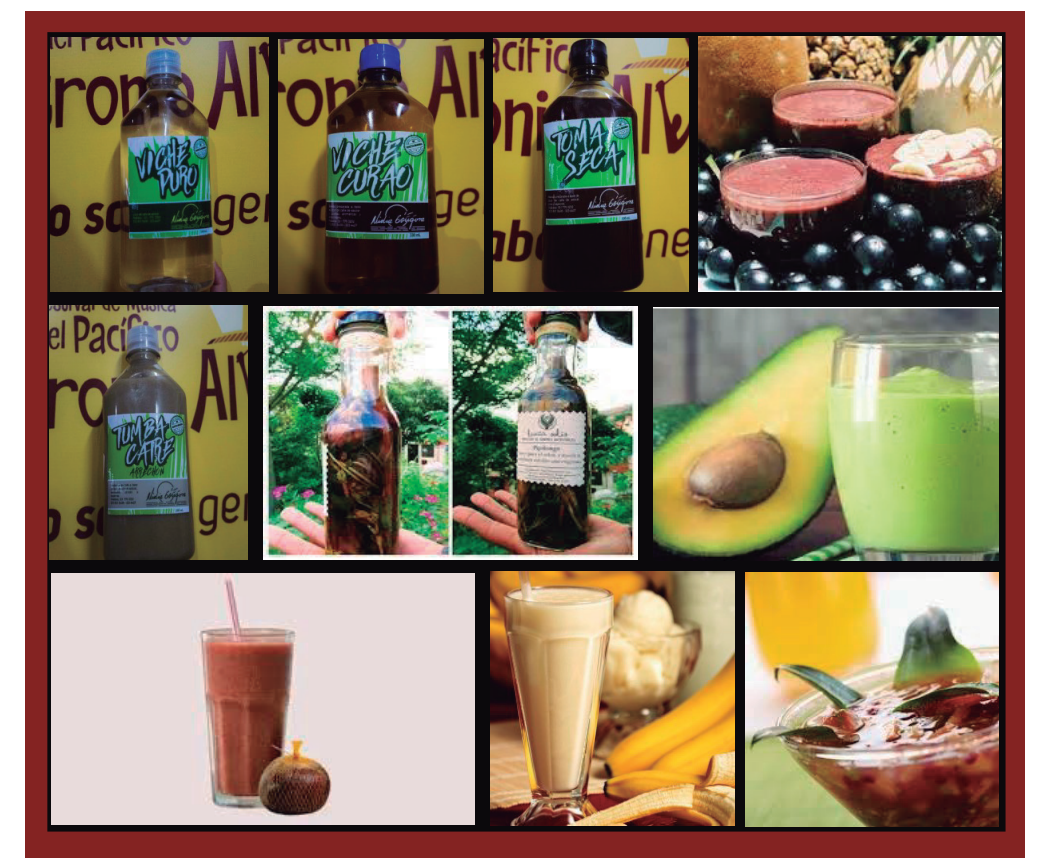

Fuente: Medardo, "clases prácticas de bebidas ancestrales", 2019. 


\section{Resultados}

Dentro de los resultados destacados en el desarrollo investigativo se evidenció que datos de trabajo en torno a las bebidas tradicionales y ancestrales de la costa pacífica no están determinados a gran escala dificultando la caracterización de las diferentes zonas de Colombia, partiendo de estos antecedentes se inicia con el proceso de generar la indagación de cada bebida para identificar el uso de los ingredientes que permitan la divulgación de las bebidas tradicionales tanto alcohólicas como no alcohólicas.

De esta manera se inicia con la organización de ponencias en diferentes espacios institucionales, en los cuales permiten gran transferencia de la información e indagación realizada, como la que se llevó a cabo por el equipo de la Fundación Universitaria San Mateo en el Festival Gastronómico de la Calera, con presencia de más 200 personas de diferentes entornos culturales y sociales, seguido a ello se genera la participación de una ponencia en el Congreso Gastronómico de Popayán, en el cual se reitera la importancia de la cultura ancestral frente a un público nacional e internacional de 500 personas.

Figura 2. Registro de participación de ponencias

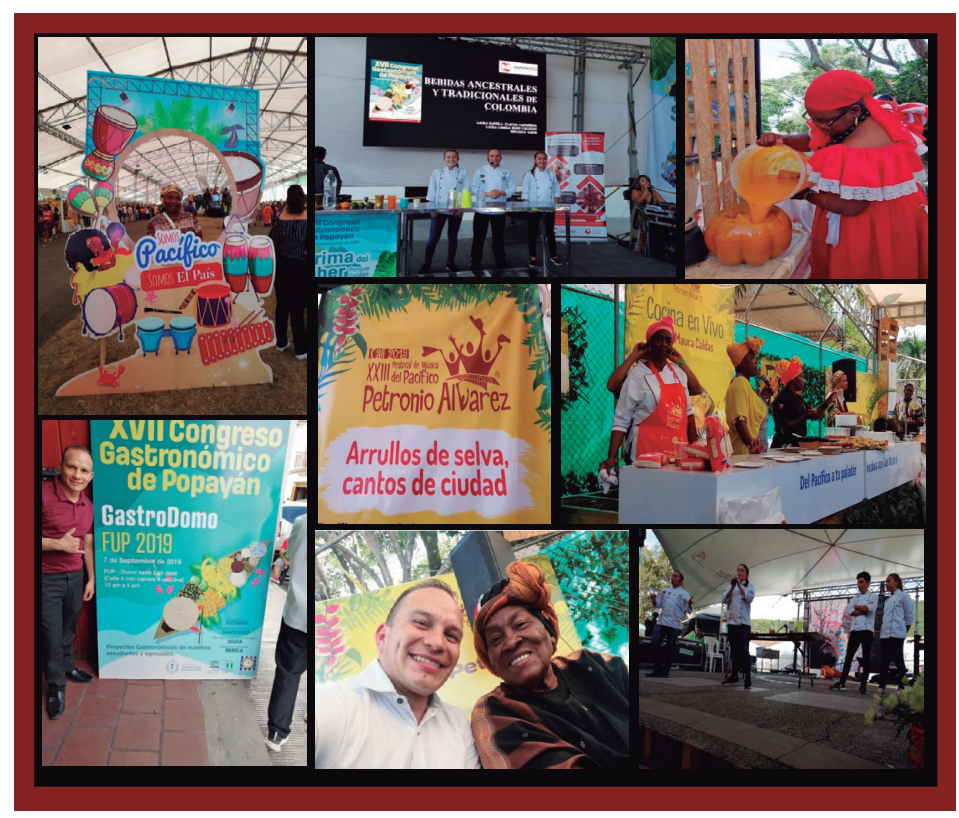

Fuente: elaboración propia. 
En este sentido, se identifican las siguientes características de preparación de las bebidas ancestrales:

\section{Viche.}

Es una bebida alcohólica o llamado aguardiente artesanal del Pacífico colombiano el cual se elabora de caña de azúcar o de panela la cual se deja fermentar y luego pasa a un proceso de destilación en alambiques artesanales a base de chorotes de barro o de aluminio adaptadas para transformarlas en elementos para generar destilación casera o artesanal en comunidades de aislamiento.

Esta bebida es de tradición cultural, medicinal, rituales funerarios y festivas, preparada por las mujeres del Valle, Chocó, Nariño y Cauca. Se ha venido preparando de generación en generación, usada por matronas junto con hierbas medicinales para curar enfermedades y a su vez se asimila con propiedades afrodisiacas.

La producción de aguardientes artesanales es introducida por los colonizadores del siglo XVII y XVIII en América y el Caribe y de allí parte el antecedente histórico de la mano de los afrodescendientes que generaban estas bebidas para su consumo, esta bebida se expande por la costa Pacífica y la costa Atlántica intercambiando ingredientes y procesos con los saberes de las diferentes etnias indígenas que los desarrollaron para su consumo y para generar ingresos en pro de una evolución económica para las comunidades (Ministerio de Cultura, 2009, p.8).

El viche se fábrica, comercializa y consume en toda la región pacifica, partiendo desde las comunidades de mayor producción como lo son poblaciones pequeñas y aisladas. Esto debido a que no cuentan con los requisitos legales de producción por ser una bebida artesanal, estas regiones López de Micai y Saija en el departamento de Cauca Sibiru, Iliza y Villamaria y en el departamento del Chocó, Triana en zona rural de buenaventura en el Valle del Cauca en donde se celebra cada año el festival del viche, así mismo encontramos los lugares de mayor comercialización y consumo como lo son poblaciones urbanas como Cali y Buenaventrura en el Valle del Cauca, Guapi en el departamento del Cauca y Pizarro en el Chocó (Colombia Patrimonio Cultural, s.f., p. 12).

Su preparación viene del sumo de la caña viche que se obtiene cuando se pasa la caña a través de los trapiches. Después se destila, proceso conocido comúnmente en calentar tal sumo para separar las sustancias que componen el mismo. 
Del viche se derivan muchas bebidas tradicionales que se elaboran y se consumen como bebedizos con propiedades medicinales y curativas, sumado a creencias de desarrollo afrodisíacos estos procesos están ligados a conocimientos ancestrales de la medicina naturista de las comunidades afrocolombianas, dentro de las más destacadas encontramos:

\section{Viche Curao.}

Su base es el viche puro y este se mezcla con una gran variedad de plantas medicinales entre ellas la menta y manzanilla. No es posible conocer a fondo los componentes de sus recetas puesto que las mismas llevan elementos que solo son conocidos por ellos y los cuales le dan el valor agregado de su sabor y apariencia, pero se encuentran algunos que representan y dan a conocer sus ingredientes para generar calidad y confianza con el consumidor.

\section{Tomaseca.}

Es una variedad de viche, solo que este es más dulce y está hecha con clavos, canela, pimienta y nuez moscada al gusto. Agregando miel para enfatizar el sabor. Este tipo de bebida es consumido en su mayoría por las mujeres que han dado a luz, con el fin de limpiar el organismo.

\section{Tumbacatre o arrechón.}

Otro tipo de bebida a base viche es conocido como el Tumbacatre o arrechón, es una preparación que se hace con los ingredientes con los que se elabora la tomaseca, adicionándole borojó y leche. Es preciso denotar que tales bebidas cambian de nombre de acuerdo con la región donde se elaboren.

\section{Pipilongo.}

Es una bebida propia del Chocó viene de la familia del curao. Se empaca en botellas curadas y contiene raíces y hiervas medicinal. Su planta principal es el Pipilongo la cual es una planta con desarrollo medicinal con trayectoria tradicional que para las comunidades genera un gran recurso tanto social como económico.

\section{Fresco de aguacate (Chocó).}

Esta bebida tradicional se consume típicamente partiendo de los nutrientes y aportes vitamínicos que aporta el aguacate, sumado al generar una bebida refrescante por las altas temperaturas que se generan en la zona del Pacifico.

En datos de indagación encontramos que el primer aguacate descubierto fue en México en la ciudad de Puebla, según estudios se cree que fue cosechado hace más de 5000 años para el consumo de las comunidades indígenas de la región 
como lo son las tribus maya, inca y olmeca, el significado del nombre aguacate es testículo que se refiere a la forma de esta fruta, también se reconoce como la fruta de la fertilidad ya que los aztecas creían que era afrodisíaca en hombre y mujeres y también les aportaba fuerza a quien lo consumiera.

\section{Jugo de naidí (Guapi cauca).}

El naidi o conocido como el açaí es el fruto de un tipo de palmera (Euterpe Oleracea) que crece exclusivamente en etapa silvestre, en zonas lluviosas y regiones húmedas cerca de los ríos, dentro del más popular y reconocido el río Amazonas, en las zonas de la amazonia se producen estos arbustos llamados acaizeiros.

Esta palmera de açaí, o como la conocen en la región del pacífico colombiano, naidy, se compone de una valla de color morado oscuro de perfil redondo, este fruto es de gran cosecha en los meses de Agosto, Septiembre y Octubre y es de gran alimento el cual se da en el municipio de Timbiquí y sus zonas aledañas. Esta región es uno de los territorios con mayor diversidad en el mundo de acuerdo con su ubicación geográfica, la cual aporta una diversidad por sus bosques húmedos cerca a los manglares y en las cabeceras de los ríos.

\section{Salpicón de baudilia.}

Esta bebida tradicional es consumida en las zonas cálidas del Chocó y en parte del Valle del Cauca partiendo de diversidad de historias y mitos de su elaboración, se representa por la mezcla de diferentes frutas de la zona junto con hielo picado, se caracteriza por el gran desarrollo de mezclas base con frutos amarillos y frutos rojos, como lo son:

\section{Mora.}

Estas frutas son oriundas de Asia y Europa y se pueden ver en estado silvestre en márgenes de caminos o torrenteras. Crecen en terrenos húmedos y en algunos casos, como el pacharán, se pueden encontrar a 1.500 metros de altitud. Maduran durante los meses de verano y otoño.

Piña.

Es una fruta tropical originaria de América del Sur. No se sabe con certeza el país donde se dio origen, pero los estudios señalan a Brasil, Paraguay y Argentina. De ahí se propagó principalmente al Amazonas, Venezuela y Perú para luego emigrar a Europa y Asia.

\section{Lulo.}

Como fruto representativo del Valle y el Chocó hace parte de esta bebida aportando sus características de sabor y sus notables aportes de sabor. 


\section{Guanábana.}

Es una planta procedente de la parte tropical de Sudamérica pero ha sido implantada en muchas regiones del Caribe en las cuales Colombia desarrolla un buen proceso en la elaboración de esta fruta.

\section{Sorbete de borojó.}

Parte de un fruto de su mismo nombre borojó el cual se representa por un color verde opaco que después de su maduración pasa a un color llamativo casi tipo chocolate, en datos encontramos que se representa normalmente con una dimensión de 7-12 centímetros de diámetro. El borojó (Borojoapatinoi) se produce en un árbol de mediano tamaño, de copa o cabezote de hoja cónica y flores tipo azahar blancas, pertenece a la familia de las rubiáceas, de la misma característica que el café.

\section{Discusión}

Es natural asociar las preparaciones de cocina en el momentos de realizar un proceso de investigación de gastronomía a nivel nacional, pero en este proceso desarrollado en torno a las bebidas se ha involucrado toda temática de las bebidas tanto alcohólicas como no alcohólicas, generando incluso un proceso de reconocimiento de los platos o alimentos de consumo casual con los cuales se elabora un maridaje tradicional de cada bebida, sumado a un gran proceso de vinculación con espacios culturales como lo son las actividades de canto y danza tradicionales de las comunidades del Valle del Cauca y del Pacifico chocoano.

De esta manera se evidencia un producto que se emplea de manera social y de manera cultural, con las cuales también se realizan tradicionalmente el consumo como producto medicinal, claro está que unificado con plantas que con sabiduría ancestral se utilizan como elementos medicinales.

\section{Conclusiones}

La representación de la cultura colombiana por medio de las bebidas es un campo de trabajo bastante amplio, partiendo de que cada fruta es base para la elaboración de una bebida y que sumado a ellos la ubicación geográfica de Colombia brinda una alta producción de múltiples frutas del trópico. 
Las bebidas alcohólicas nacionales parten de un desarrollo ancestral como lo son los fermentados y los destilados. En la región Pacifica se da nacimiento a la primera bebida destilada artesanalmente que seguido a ello se expande por el país y genera una gran diversificación y afluencia de consumo a nivel nacional.

Como contexto y trabajo para la apropiación de productos y preparaciones nacionales en pro de generar un reconocimiento a esos saberes. Esta investigación a generado gran expectativa en la comunidad educativa y en los diferentes espacios de discusión gastronómica se brinda la oportunidad de dar la relevancia que se les debe a estas bebidas y productos.

\section{Referencias bibliográficas}

Chaves-López, C. (2014). Traditional fermented foods and beverages from a microbiological and nutritional perspective: The Colombian heritage. Comprehensive Reviews in Food Science and Food Safety, 13(5), pp. 1031-1048.

Colombia patrimonio cultural. (s.f.). Región Pacífica. Colombia patrimonio cultural. Recuperado de https://colombiapatrimoniocultural.wordpress.com/ region-pacifica/

Granados, C. (2013). Evaluación fisicoquímica y microbiológica del aperitivo vínico de lulo (Solanum quitoense L.), Información Tecnológica, 24(6), pp. $35-40$.

Ministerio de Cultura. (2013). Política para el conocimiento, la salvaguardia y el fomento de la alimentación y las cocinas tradicionales en Colombia. Bogotá: Ministerio de Cultura. Recuperado de https://www.mincultura. gov.co/ministerio/politicas-culturales/Pol\%C3\%ADtica\%20de\%20las\%20 cocinas\%20tradicionales\%20de\%20Colombia/Paginas/default.aspx 